\title{
THE LATE ALBIAN-EARLY CENOMANIAN OSTRACODES FROM NAUR FORMATION, JORDAN
}

\author{
CRISTIANINI TRESCASTRO BERGUE, GERSON FAUTH \\ Universidade do Vale do Rio dos Sinos, itt Fossil, Av. Unisinos 950, 93022-750, São Leopoldo, RS, Brasil. \\ ctbergue@gmail.com,gersonfauth@gmail.com \\ JOÃO CARLOS COIMBRA \\ Instituto de Geociências, UFRGS, Av. Bento Gonçalves 9500, 91501-970, Porto Alegre, RS, Brasil. \\ joao.coimbra@ufrgs.br
}

FAYEZ YACOB AHMAD, AHMAD SMADI

Department of Environment and Earth Sciences, Hashemite University, Zarqa, Jordan.

SHERIF FAROUK

Egyptian Petroleum Research Institute, Nasser City 11727, Cairo, Egypt.

\begin{abstract}
Thirty-nine species of podocopid and platycopid ostracodes were recovered from the well NH-1, in AlKhaldeh, Jordan. Typical Cenomanian taxa such as Metacytheropteron berbericus Bassoulet \& Damotte, Cytherella aegyptiensis Colin \& El Dakkak, Cythereis namousensis Bassoulet \& Damotte, Cythereis algeriana Bassoulet \& Damotte, Veeniacythereis jezzineensis Bischoff and Dolocytheridea atlasica Bassoulet \& Damotte, common in other South Tethyan regions, were recorded. Pore-clusters, a typical Eucytherurinae morphological feature, are recorded in Aracajuia distincta (Gerry \& Rosenfeld).
\end{abstract}

Key words: Tethys, paleozoogeography, taxonomy, Cretaceous, pore-clusters.

RESUMO - Trinta e nove espécies de ostracodes podocopídeos e platicopídeos foram recuperadas do poço NH-1, em AlKhaldeh, Jordânia. Táxons tipicamente cenomanianos como Metacytheropteron berbericus Bassoulet \& Damotte, Cytherella aegyptiensis Colin \& El Dakkak, Cythereis namousensis Bassoulet \& Damotte, Cythereis algeriana Bassoulet \& Damotte, Veeniacythereis jezzineensis Bischoff e Dolocytheridea atlasica Bassoulet \& Damotte, comuns em outras regiões do sul do Tétis foram registrados. Grupos de poros, uma característica morfológica típica dos Eucytherurinae, são observadas em Aracajuia distincta (Gerry \& Rosenfeld).

Palavras-chave: Tétis, paleozoogeografia, taxonomia, Cretáceo, grupos de poros.

\section{INTRODUCTION}

The Cretaceous ostracodes from Southern Tethys have already been extensively studied, revealing some stratigraphical markers (e.g. Veeniacythereis jezzineensis, Metacytheropteron berbericus, Cythereis algeriana and Dolocytheridea atlasica) useful in some localities (Grosdidier, 1973; Rosenfeld \& Raab, 1974; Majoran, 1989; Athersuch, 1994). Paleobiogeographic analyses allowed also the proposal of faunal provinces for the Aptian and younger deposits (Babinot \& Colin, 1988; Luger, 2003).

In spite of that, there are few published studies on Cenomanian ostracodes of Jordan. Babinot \& Basha (1985) registered 17 species from six sections of Naur and Fuhies formations (early Cenomanian), east of the Jordan Rift Valley. They were the first to discuss the similarity of those faunas to others of the Tethyan realm. Powell (1989) in his study on the groups Kurnub, Ajlun and Belqa mentioned the occurrence of five species in the Naur Formation: Metacytheropteron berbericus, Veenia jezzineensis, Schuleridea sp., Bythocypris sp. and Cytherura sp. Later, Schultze et al. (2004) detailed the paleoecology and biostratigraphy of the Jordanian carbonate platform based on ostracodes and foraminifers, which improved the understanding of the shelf development during the late Albian-Turonian. More recently, Morsi \& Wendler (2010) carried out a study on the Cenomanian-Turonian deposits of Jordan discussing not only biostratigraphical aspects, but also parameters influencing the ostracode distribution.

The main objective of this study is, therefore, to present an updated contribution to the knowledge of the late Albian-early Cenomanian ostracodes from Naur Formation. The specimens 
herein illustrated are deposited in the collections of the Museu de Paleontologia, Universidade Federal do Rio Grande do Sul, section "Ostracoda" (MP-O), under the curatorial numbers 2201 to 2233 and 2565 to 2582. Morphological abbreviations: $\mathbf{C}$, carapace; $\mathbf{R V}$, right valve; $\mathbf{L V}$, left valve; $\mathbf{l}$, length; $\mathbf{h}$, height; $\mathbf{w}$, width.

\section{STUDY AREA}

In Jordan, thick Upper Cretaceous to Paleogene successions crop out over a wide area. These successions are subdivided into two groups: the upper Albian-Turonian Ajlun Group and the Coniacian to Paleogene Belqa Group. The Ajlun Group is further subdivided into the Naur (base), Fuheis, Hummar, Shuayb, and Wadi As Sir formations (Burdon, 1959; Parker, 1970; Powell, 1989).

During the Cenomanian and Turonian stages, shallow warm seas covered Jordan depositing the strata of the Ajlun Group. The Naur Formation consists of clayed siltstone, sandstone, silty mudstone, marls, dolomites and limestone with abundant Thalassinoides burrows. The Fuheis Limestone is dominated by marl and marly limestone. The Hummar Formation is dominated by limestone, micrite and shelly wackestone. The Shuayb Formation is overlain by the limestones of the Wadi As Sir Formation. The Shuayb Formation consists of a rhythmic alternation of thinly bedded limestones and mostly fossiliferous marly limestones. The overall composition of this formation becomes totally marly towards its top (Powell, 1989).

The mostly marine Ajlun Group overlies the Lower Cretaceous Kurnub throughout most of the territory. The boundary between Kurnub and Ajlun groups is diachronous, marking the transition from terrestrial to marine environments. According to benthic foraminifera data these deposits correspond to a transgression of the north and northwest parts of Tethys Ocean during the Albian-early Cenomanian (Basha, 1985).

\section{MATERIAL AND METHODS}

The ditch cutting samples here studied are from the well NH-1 drilled in Al-Khaldeh north-northeastern Jordan (Figure 1), between the coordinates $32^{\circ} 12^{\prime} 21^{\prime \prime} \mathrm{N}$; $36^{\circ} 13^{\prime} 58^{\prime \prime}$. They were collected from 330-50 m depth and treated for their faunal content with hydrogen peroxide bath (for disaggregation) and water washing (for sieving). The occurrence of species previously recorded in other South Tethyan localities, such as Veeniacythereis jezzineensis (Bischoff, 1963), Dolocytheridea atlasica Bassoulet \& Damotte, 1969, Metacytheropteron berbericum (Bassoulet \& Damotte, 1969), Cythereis algeriana Bassoulet \& Damotte, 1969, C. namousensis Bassoulet \& Damotte, 1969 and Cytherella gigantosulcata Rosenfeld, 1981, give support to a late Albian-Cenomanian age for the strata herein studied.

Representative specimens of each species were selected for imaging in SEM. The suprageneric classification follows Liebau (2005) and the generic one is based mostly on Benson et al. (1961).

\section{SYSTEMATIC PALEONTOLOGY}

Subclass OSTRACODA Latreille, 1802

Superorder PODOCOPOMORPHA Kozur, 1972

Order PLATYCOPIDA Sars, 1866

Superfamily CYTHERELLOIDEA Sars, 1866

Family CYTHERELLIDAE Sars, 1866

Cytherella Jones, 1849

Type species. Cytherina ovata Roemer, 1841.

Cytherella aegyptiensis Colin \& El Dakkak, 1975

(Figures 2A-E)
A

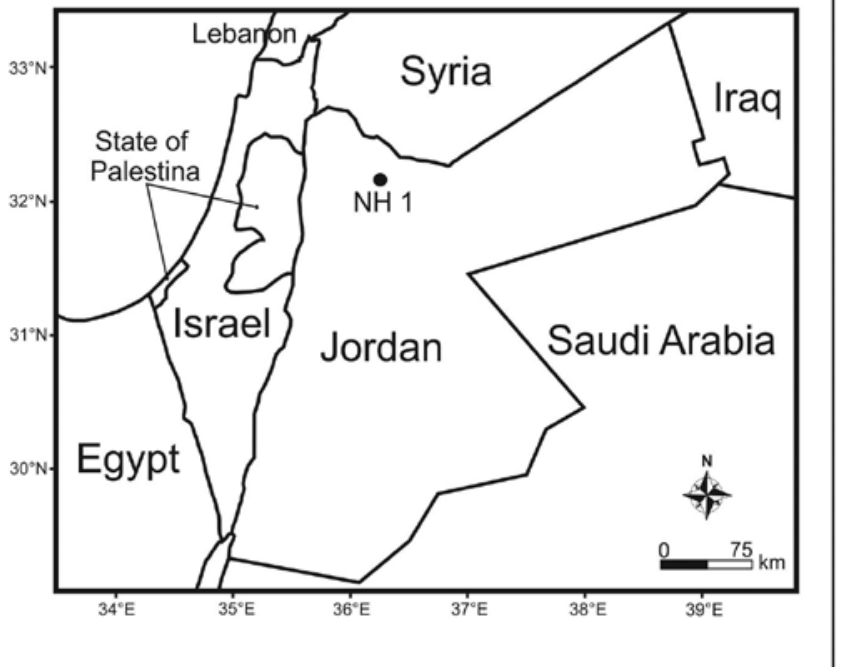

B
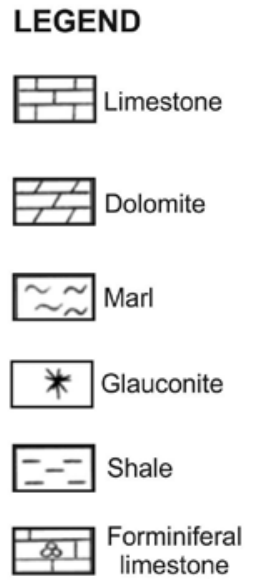

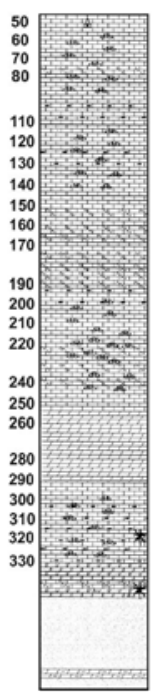

Figure 1. A, Study area with the location of the well $\mathrm{NH}-1$; B, The well $\mathrm{NH}-1$ log with the respective sampling depths (m). 
1975 Cytherella aegyptiensis Colin \& El Dakkak, p. 50, pl. 1, figs. 2, 3.

1991 Cytherella aegyptiensis Colin \& El Dakkak. Szczechura et al., pl. 1, figs. 1-6.

Figured specimens. MP-O-2201, female C, 1: $0.96 \mathrm{~mm}$, h: $0.65 \mathrm{~mm}$, w: $0.51 \mathrm{~mm}$; MP-O-2202, male C, $1: 0.93 \mathrm{~mm}$, h: $0.54 \mathrm{~mm}$, w: $0.47 \mathrm{~mm}$; MP-O-2203, female RV, $1: 1.0 \mathrm{~mm}$, h: $0.70 \mathrm{~mm}$; MP-O-2204, female LV, 1: $0.89 \mathrm{~mm}$, h: $0.54 \mathrm{~mm}$. Origin. sample $250 \mathrm{~m}$ (MP-O-2201/02), sample $60 \mathrm{~m}$ (MPO-2203), sample $220 \mathrm{~m}$ (MP-O-2204).

Material. 481 specimens.

\section{Cytherella gigantosulcata Rosenfeld, 1981}

(Figures 2F-G)

1973 Cytherella U-10 Glintzboeckel \& Magné. Grosdidier, p. 141, pl. 1, figs. 2a-c.

1974 Cytherella sulcata Rosenfeld, pl. 1, figs. 6-8.

1981 Cytherella sulcata Rosenfeld. Bismuth et al., p. 223, pl. 6, figs. 3 and 4.

1981 Cytherella sulcata Rosenfeld. Al-Abdul-Razzaq \& Grosdidier, p. 181, pl. 1, fig. 1.

1988 Cytherella posterosulcata Rosenfeld. Athersuch, p. 202, pl. 5, fig. 1.

1991 Cytherella gigantosulcata Rosenfeld. Szczechura et al., pl. 1, figs. 7-12 (see this for a more complete synonymic list).

Figured specimen. MP-O-2205, C, 1: $0.72 \mathrm{~mm}$, h: $0.58 \mathrm{~mm}$, w: $0.42 \mathrm{~mm}$.

Origin. sample $240 \mathrm{~m}$.

Material. Two specimens.

Remarks. C. gigantosulcata is a typical Cenomanian species described in Hamakhtesh Hagadol (Israel), easily identified by the well developed sulcus in the RV. It is rare in the material studied and occurs only in the sample NH1 $240 \mathrm{~m}$.

\section{Cytherella dhalalensis Morsi \& Bauer, 2001}

(Figures 2H-I)

?1991 Cytherella cf. eosulcata Colin. Szczechura et al., pl. 2, fig. $7 \mathrm{a}$.

2001 Cytherella dhalalensis Morsi \& Bauer, p. 399, pl. 1, figs. 3-5.

2010 Cytherella dhalalensis Morsi \& Bauer. Morsi \& Wendler, p. 194, fig. 5.4-5.

Figured specimen. MP-O-2206, C, 1: $0.77 \mathrm{~mm}$, h: $0.42 \mathrm{~mm}$, w: $0.33 \mathrm{~mm}$.

Origin. Sample $260 \mathrm{~m}$.

Material. 21 specimens.

Remarks. This species, described in the Sinai Peninsula (Egypt) is common in Cenomanian deposits of South Tethyan region.

Cytherella sp. 1

(Figures 2J-K)
Figured specimen. MP-O-2207, C, 1: $0.89 \mathrm{~mm}, \mathrm{~h}: 0.51 \mathrm{~mm}$, w: $0.40 \mathrm{~mm}$.

Origin. Sample $110 \mathrm{~m}$.

Material. One C.

Remarks. Cytherella sp. 1 is very rare in the material studied and occurs only in the sample $110 \mathrm{~m}$. Puckett et al. (2012) recorded a similar species identified as Cytherella tuberculifera Alexander in the Maastrichtian of Jamaica. Honigstein (1984) found a Cytherella species with a median caudal process which was identified as $C$. coryelli Benson \& Tatro, 1964, in Santonian/Campanian deposits of Israel.

Cytherella sp. 2

(Figures 2L-M)

1991 Cytherella sp. cf. Cytherella IRC 6 Grosdidier. Szczechura et al., pl. 2, figs. 1-6.

Figured specimen. MP-O-2208, C, 1: $0.96 \mathrm{~mm}, \mathrm{~h}: 0.61 \mathrm{~mm}$, w: $0.49 \mathrm{~mm}$.

Origin. Sample $60 \mathrm{~m}$.

Material. 16 specimens.

Remarks. Cytherella sp. 2 is similar to Cytherella aegyptiensis but differ in the outline of the anterior margin, which is more broadly rounded, while in C. aegyptiensis it is slightly more acute.

\author{
Order PODOCOPIDA Sars, 1866 \\ Suborder BAIRDIOCOPINA Gründel, 1967 \\ Superfamily BAIRDIOIDEA Sars, 1887 \\ Family BAIRDIIDAE Sars, 1887
}

Bairdia McCoy, 1844

Type species. Bairdia curtus McCoy 1844

Remarks. Coryell et al. (1935) proposed the genus Bairdoppilata based on the assumption that such a conservative form as Bairdia would be unusual among the arthropods, due to its long stratigraphic range. The genus erected by them is characterized by the presence of transverse teeth in the hingement. Later, Maddocks (1969) in a revision of the recent Bairdiidae reinforced the reasoning that the genus Bairdia should be restricted to the Paleozoic. According to her, the carapace morphology would be enough for the distinction between Bairdia and Bairdoppilata, although this statement partly disagrees with the remark on page 68 of the same work, where that author argues that the data on soft parts anatomy are congruent "or even more consistent than the hinge composition" of Bairdoppilata. Moreover, besides the hingement composition, no other carapace characters are listed as diagnostic for either Bairdia or Bairdoppilata. What makes this assumption even more questionable is the occurrence of denticles in Paleozoic species (= bairdoppilate denticles sensu Liebau, 2005).

We agree with the statements of both Maddocks (1969) and Coryell et al. (1935) that Bairdia is an overextended, and, probably, not a natural taxa. A similar pattern has been demostrated by the revision of the genus Darwinula Brady \& 
Robertson, 1885 for instance, which resulted in the proposal of a few other genera (Rossetti \& Martens, 1998). However, we do not believe that the criteria presented by Maddocks (1969) would be satisfactorily applied to all species of Bairdia/Bairdoppilata without previous knowledge of the soft anatomy and the chronostratigraphic distribution of a species. The distinction of these two genera based solely on the denticles is not adequate for fossil species, and can promote more confusion to the ostracode taxonomy. Therefore, in the present study both taxa are considered synonym and the species assigned to the genus Bairdia.

Bairdia youssefi Bassiouni, 2002

(Figures 2N-P)

2002 Bairdia youssefi Bassiouni, pl. 1, figs. 1, 3.

2010 Bairdia youssefi Bassiouni. Morsi \& Wendler, p. 194, fig. 5.10 .

Figured specimens. MP-O-2209, female C, l: $0.93 \mathrm{~mm}$; h: $0.63 \mathrm{~mm}$, w: $0.54 \mathrm{~mm}$; MP-O-2210, male C, 1: $0.93 \mathrm{~mm}$, h: $0.58 \mathrm{~mm}$, w: $0.49 \mathrm{~mm}$.

Origin. Sample $200 \mathrm{~m}$.

Material. 38 specimens.

Remarks. Bairdia youssefi is similar to Bairdia sp. (T-378) illustrated by Rosenfeld \& Raab (1974), however, differs in the outline of posterior margin.

\section{Bairdia sp. 1}

(Figures 2Q-R)

Figured specimen. MP-O-2211, C, 1: $1.0 \mathrm{~mm}$; h: $0.84 \mathrm{~mm}$, w: $0.47 \mathrm{~mm}$.

Origin. Sample $130 \mathrm{~m}$.

Material. Two specimens.

Bairdia sp. 2

(Figures 3A-B)

Figured specimen. MP-O-2212, C, 1: $1.0 \mathrm{~mm}$; h: $0.58 \mathrm{~mm}$, w: $0.51 \mathrm{~mm}$.

Origin. Sample $200 \mathrm{~m}$.

Material. 14 specimens.

Bairdia sp. 3

(Figures 3C-D)

Figured specimen. MP-O-2213, C, 1: $1.3 \mathrm{~mm}$; h: $0.63 \mathrm{~mm}$, w: $0.49 \mathrm{~mm}$.

Origin. Sample $130 \mathrm{~m}$.

Material. One specimen.

Suborder CYPRIDOCOPINA Jones, 1901

Superfamily CYPRIDOIDEA Baird, 1845

Family CANDONIDAE Kaufmann, 1900
Type species. Candoniella suzini Schneider, 1956

Remarks. According to Benson et al. (1961) Candoniella has a questionable status. No internal morphological details were observed in the specimens herein studied, which would contribute to the precise taxonomic assignement of these species.

\section{Candoniella? sp. 1}

(Figures 3E-F)

Figured specimen. MP-O-2214, C, 1: $0.82 \mathrm{~mm}$, h: $0.44 \mathrm{~mm}$, w: $0.30 \mathrm{~mm}$.

Origin. Sample $300 \mathrm{~m}$.

Material. Four specimens.

\section{Candoniella? sp. 2}

(Figures 3G-H)

Figured specimen. MP-O-2215, C, 1: $0.82 \mathrm{~mm}$, h: $0.44 \mathrm{~mm}$, w: $0.40 \mathrm{~mm}$.

Origin. Sample $300 \mathrm{~m}$.

Material. Three specimens.

Remarks. Candoniella? sp. 2 has the maximum height in the middle part of the carapace while in the Candoniella? sp. 1 it is positioned slightly backward.

\section{Candoniella? sp. 3}

(Figures 3I-J)

1991 Candoniella cf. qeturaensis Honigstein \& Rosenfeld. Szczechura et al., pl. 8, fig. 11.

Figured specimen. MP-O-2216, C, 1: $0.86 \mathrm{~mm}$, h: $0.47 \mathrm{~mm}$, w: $0.42 \mathrm{~mm}$.

Origin. Sample $320 \mathrm{~m}$.

Material. Four specimens.

Remarks. Candoniella? sp. 2 and Candoniella? sp. 3 differ in the curvature of the posterodorsal, dorsal and anterodorsal margins, which seem to be more evenly rounded in the latter. Szczechura et al. (1991) recorded this species in early/mid Cenomanian deposits of the locality Ras el Abd (Galala Plateau, Egypt).

\section{Paracypris Sars, 1866}

Type species. Paracypris polita Sars, 1866

Paracypris dubertreti Damotte \& Saint-Marc, 1972

(Figures 3K-L)

Figured specimen. MP-O-2217, C, 1: $0.70 \mathrm{~mm}$; h: $0.26 \mathrm{~mm}$, w: $0.19 \mathrm{~mm}$.

Origin. Sample $220 \mathrm{~m}$.

Material. Six specimens.

Remarks. $P$. dubertreti was described in the Middle Cenomanian of Nahr Ibrahim (Lebanon). 


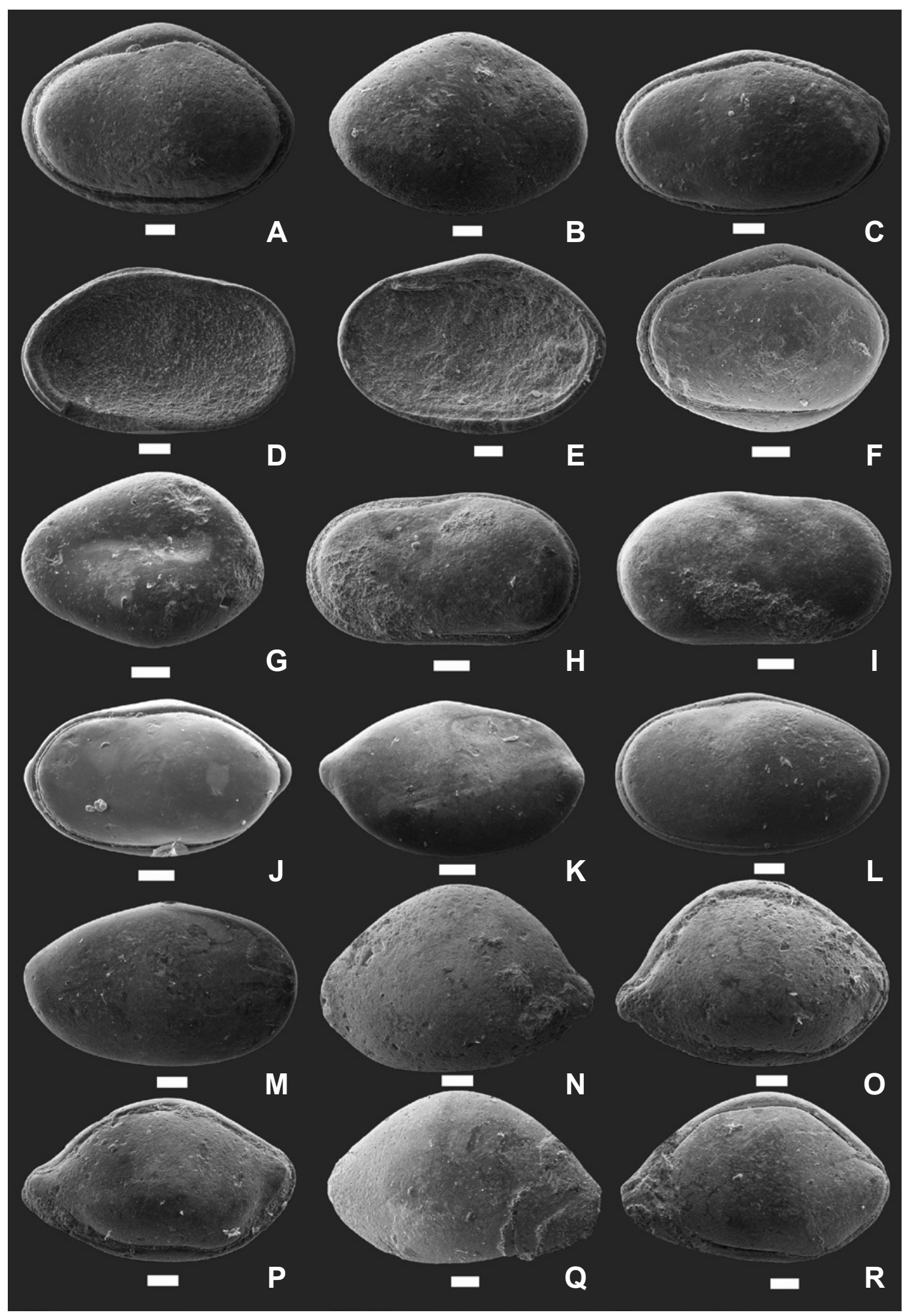

Figure 2. A-E, Cytherella aegyptiensis, A, female C left view (MP-O-2201); B, same specimen right view; C, male C left view (MP-O-2202); D, female LV internal view (MP-O-2204); E, female RV internal view (MP-O-2203). F-G, Cytherella gigantosulcata, F, C left view (MP-O-2205); G, same specimen right view. H-I, Cytherella dhalalensis, H, C left view (MP-O-2206); I, same specimen right view. J-K, Cytherella sp. 1, J, C left view (MP-O-2207); K, same specimen right view. L-M, Cytherella sp. 2, L, C left view (MP-O-2208); M, same specimen right view. N-P, Bairdia youssefi, N, female C left view (MP-O-2209); O, same specimen right view; P, male C right view (MP-O-2210). Q, Bairdia sp. 1, C left view (MP-O-2211); $\mathbf{R}$, same specimen right view. Scale bars $=100 \mu \mathrm{m}$. 
Figured specimen. MP-O-2218, C, 1: $0.82 \mathrm{~mm}$; h: $0.37 \mathrm{~mm}$, w: $0.35 \mathrm{~mm}$.

Origin. Sample $260 \mathrm{~m}$.

Material. 117 specimens.

Paracypris sp. 2

(Figures 3O-P)

Figured specimen. MP-O-2219, C, 1: $0.82 \mathrm{~mm}$; h: $0.37 \mathrm{~mm}$, w: $0.33 \mathrm{~mm}$.

Origin. Sample $310 \mathrm{~m}$.

Material. Three specimens.

Paracypris sp. 3

(Figures 3Q-R)

Figured specimen. MP-O-2220, C, 1: $0.82 \mathrm{~mm}$; h: $0.37 \mathrm{~mm}$, w: $0.35 \mathrm{~mm}$.

Origin. Sample $310 \mathrm{~m}$.

Material. Five specimens.

Suborder CYTHEROCOPINA Gründel, 1967

Superfamily XESTOLEBERIDOIDEA Sars, 1928

Family XESTOLEBERIDIDAE Sars, 1928

Xestoleberis Sars, 1866

Type species. Cythere aurantia Baird, 1838.

\section{Xestoleberis sp. 1}

(Figures 4A-B)

Figured specimens. MP-O-2221, C, 1: $0.49 \mathrm{~mm}$, h: $0.30 \mathrm{~mm}$, w: $0.35 \mathrm{~mm}$.

Origin. Sample $210 \mathrm{~m}$.

Material. 10 specimens.

\section{Xestoleberis sp. 2}

(Figures 4C-D)

Figured specimens. MP-O-2222, C, 1: $0.63 \mathrm{~mm}, \mathrm{~h}: 0.30 \mathrm{~mm}$, w: $0.30 \mathrm{~mm}$.

Origin. Sample $150 \mathrm{~m}$.

Material. One specimen.

Infraorder NOMOCYTHERININA Liebau, 1991

Superfamily CYTHEROIDEA Baird, 1850

Family CYTHERIDAE Baird, 1850

Subfamily SCHIZOCYTHERINAE Mandelstam, 1959

Aracajuia Krömmelbein, 1967 emend. Antonietto et al., 2013

Type species. Aracajuia benderi Krömmelbein, 1967

Aracajuia distincta (Gerry \& Rosenfeld, 1973)

(Figures 4E-H)
1973 Amphicytherura distincta Gerry \& Rosenfeld, pl. 2, figs. 7-10.

2013 Aracajuia distincta (Gerry \& Rosenfeld). Antonietto, Abrahão, Do Carmo \& Meirelles, p. 9, fig. 5G.

Figured specimen. MP-O-2223, C, 1: $0.40 \mathrm{~mm}, \mathrm{~h}: 0.21 \mathrm{~mm}$, w: $0.16 \mathrm{~mm}$.

Origin. sample $110 \mathrm{~m}$.

Material. Three specimens.

Remarks. A. distincta was described in Cenomanian deposits of Israel. One of the specimens herein studied presents peculiar pore clusters (see "Results and discussion" section).

Family CYTHERURIDAE Müller, 1894

Metacytheropteron Oertli, 1957

Type species. Metacytheropteron elegans Oertli, 1957

Metacytheropteron berbericus (Bassoulet \& Damotte, 1969)

(Figures 4J-K)

1969 Cytheropteron berbericus Bassoulet \& Damotte, p. 143, pl. 2, figs. 7a-d.

2009 Metacytheropteron berbericum (Bassoulet \& Damotte). Boukhary et al., p. 23, pl. 1, figs. 16-20 (see this for a complete synonymy).

Figured specimen. MP-O-2224, C, 1: $0.86 \mathrm{~mm}$, h: $0.33 \mathrm{~mm}$, w: $0.28 \mathrm{~mm}$.

Origin. Sample $320 \mathrm{~m}$.

Material. 19 specimens.

Remarks. M. berbericus is a typical Cenomanian species described in Algeria and widespread in the South Tethyan domain.

\section{Metacytheropteron sp.}

(Figures 4L-M)

1991 Metacytheropteron IRC 10 Szczechura et al., pl. 4, fig. 5.

Figured specimen. MP-O-2225, C, 1: $0.51 \mathrm{~mm}, \mathrm{~h}: 0.33 \mathrm{~mm}$, w: $0.35 \mathrm{~mm}$.

Origin. Sample $320 \mathrm{~m}$.

Material. Three specimens.

$$
\text { Eocytheropteron Alexander, } 1933
$$

Type species. Cytheropteron bilobatum Alexander, 1929

Eocytheropteron retroversicardinatum Al-Abdul-Razzaq, 1980

(Figures 4N-O)

Figured specimen. MP-O-2226, C, 1: $0.49 \mathrm{~mm}, \mathrm{~h}: 0.28 \mathrm{~mm}$, w: $0.33 \mathrm{~mm}$.

Origin. Sample $250 \mathrm{~m}$. 


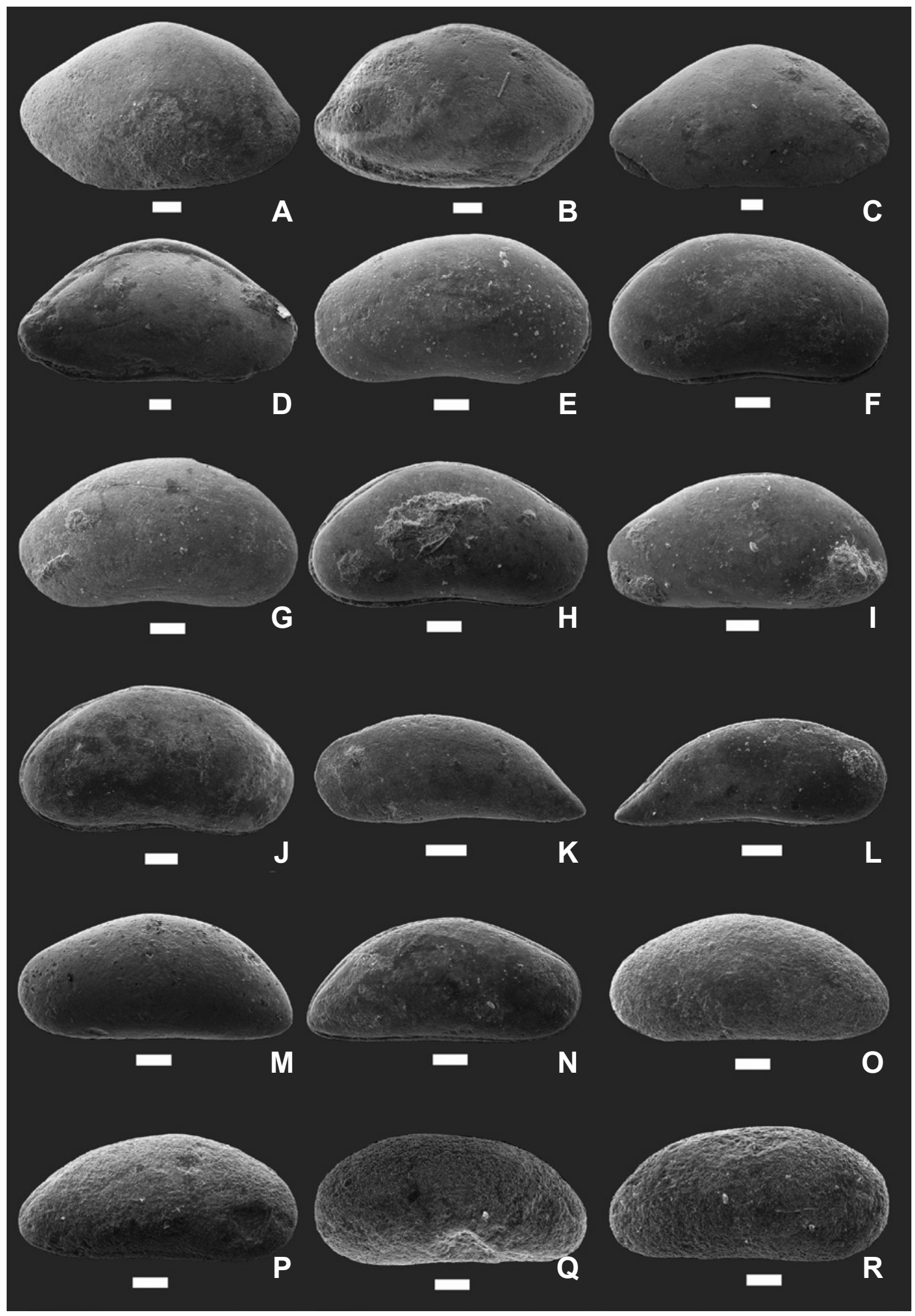

Figure 3. A-B, Bairdia sp. 2, A, C left view (MP-O-2212); B, same specimen right view. C-D, Bairdia sp. 3, C, C left view (MP-O-2213); D, same specimen right view. E-F, Candoniella? sp. 1, E, C left view (MP-O-2214); F, same specimen right view. G-H, Candoniella? sp. 2, G, C left view (MP-O-2215); H, same specimen right view. I-J, Candoniella? sp. 3, I, C left view (MP-O-2216); J, same specimen right view. K-L, Paracypris dubertreti, K, C left view (MP-O-2217); L, same specimen right view. M-N, Paracypris sp. 1, M, C left view (MP-O-2218); N, same specimen right view. O-P, Paracypris sp. 2, O, C left view (MP-O-2219); P, same specimen right view. Q-R, Paracypris sp. 3, Q, C left view (MP-O-2220); $\mathbf{R}$, same specimen right view. Scale bars $=100 \mu \mathrm{m}$. 
Material. Nine specimens.

Remarks. This species was described in the early Cenomanian Ahmadi Limestone (Kuwait). In the type-locality it was noticed that some specimens presented valve reversion.

\section{Superfamily TRACHYLEBERIDOIDEA Sylvester-Bradley, 1948}

Family TRACHYLEBERIDIDAE Sylvester-Bradley, 1948 Subfamily TRACHYLEBERIDINAE Sylvester-Bradley, 1948

Sergipella Krömmelbein, 1967 emend. Do Carmo et al., 2012

Type species. Sergipella transatlantica Krömmelbein, 1967

Sergipella sp.

(Figures 4P-R)

Figured specimens. MP-O-2227, female C, 1: $0.61 \mathrm{~mm}, \mathrm{~h}$ : $0.35 \mathrm{~mm}$, w: $0.28 \mathrm{~mm}$; MP-O-2228, male C, $1: 0.63 \mathrm{~mm}$, h: $0.33 \mathrm{~mm}, \mathrm{w}: 0.30 \mathrm{~mm}$.

Origin. sample $310 \mathrm{~m}$.

Material. Three specimens.

Remarks. This species is similar to Hechticythere croutensis (Damote \& Grosdidier, 1963) recorded in the late Aptian of Sinai (Egypt) by Bassiouni (2002). However, based on the work by Do Carmo et al. (2012) we believe that it fits better into the emended diagnosis of Sergipella.

\section{Veeniacythereis Gründel, 1973}

Type species. Cythereis imparia Gründel, 1968

Veeniacythereis jezzineensis (Bischoff, 1963)

(Figures 5A-D)

1963 Cythereis jezzineensis Bischoff, p. 42, pl. 16, figs. 128-130. 1969 Cythereis maghrebensis Bassoulet \& Damotte, p. 141, pl. 1, figs. 2a-c.

1981 Veeniacythereis jezzineensis (Bischoff, 1963). Al-AbdulRazzaq \& Grosdidier, p. 181, pl. 1, fig. 2.

1981 Veeniacythereis maghrebensis (Bassoulet \& Damotte). Al-Abdul-Razzaq \& Grossdidier, p. 181, pl. 1, fig. 3.

1981 Cythereis streblolophata streblolophata Al-AbdulRazzaq \& Grosdidier, p. 181, pl. 1, figs. 7-10; p. 187, pl. 2, figs. 6-8.

1981 Cythereis streblolophata schista Al-Abdul-Razzaq \& Grosdidier, p. 187, pl. 2, figs. 1-5.

1983 Veeniacythereis jezzineensis (Bischoff, 1963). Rosenfeld \& Raab, pl. 1, figs. 1-15; pl. 2, figs. 1-13.

1988 Veeniacythereis subrectangulata Majoran, p. 693, pl. 1, figs. 1-15; pl. 2, figs. 1-8.

1988 “Veeniacythereis" sp. nov.? Majoran, p. 695, pl. 3, figs. 1-7. 1988 Veeniacythereis aff. streblolophata. Majoran, p. 695, pl.3, figs. 8-10.

1991 Veeniacythereis jezzineensis (Bischoff, 1963). Szczechura et al., pl. 7, figs. 4, 9-11.
1991 Veeniacythereis ex gr. streblolophata. Szczechura et al., pl. 7, figs. 1-3, 5-8.

2002 Veeniacythereis jezzineensis (Bischoff, 1963). Bassiouni, pl. 19, figs. 1-7.

2002 Veeniacythereis maghrebensis (Bassoulet \& Damotte). Bassiouni, pl. 19, figs. 8-11.

2002 Veeniacythereis streblolophata schista (Al-AbdulRazzaq \& Grosdidier). Bassiouni, pl. 18, figs. 13-16; pl. 19, fig. 12.

2009 Veeniacythereis streblolophata schista (Al-AbdulRazzaq \& Grosdidier). Boukhary et al., p. 27, pl. 2, figs. 11-15. 2010 Veeniacythereis maghrebensis (Bassoulet \& Damotte). Morsi \& Wendler, p. 200, figs. 7.9-11,13.

2010 Veeniacythereis streblolophata schista (Al-AbdulRazzaq \& Grossdidier). Morsi \& Wendler, p. 200, figs. 7.12, $14,15$.

Figured specimens. MP-O-2229 adult C, 1: $0.93 \mathrm{~mm}$, h: $0.58 \mathrm{~mm}$, w: $0.54 \mathrm{~mm}$; MP-O-2230 A-1(?) C, $1: 0.79 \mathrm{~mm}$, h: $0.44 \mathrm{~mm}$; MP-O-2231 A-2(?) C, $1: 0.77 \mathrm{~mm}$, h: $0.42 \mathrm{~mm}$, w: $0.42 \mathrm{~mm}$.

Origin. Sample $150 \mathrm{~m}$.

Material. 109 specimens.

Remarks. Veeniacythereis jezzineensis is a typical late Albian-Cenomanian species described in Jezzine, Lebanon. According to Rosenfeld \& Raab (1983), Veeniacythereis streblolophata streblolophata (Al-AbdulRazzak \& Grosdidier, 1981) and V. s. schista (Al-AbdulRazzaq \& Grosdidier, 1981) are instars (A-1 to A-3) of V.jezzineesis. The species Cythereis maghrebensis Bassoulet \& Damotte, 1969, Veeniacythereis subrectangulata Majoran, 1988, "Veeniacythereis" sp. nov.? Majoran, 1989 and V. streblolophata tunisiaensis Majoran, 1996 are also junior synonyms of V.jezzineensis. Colin \& El Dakkak (1975) were the first authors who considered the streblolophata morphs as juveniles of jezzineensis.

Viviére (1985), Athersuch (1994) and Colin et al. (2001) follow this synonymy. But it is not accepted by Majoran (1996) who claims that "this assignation is improbable since Veeniacythereis streblolophata displays a fully developed amphidont hinge and sexual dimorphisms which are characters usually associated with adult forms". Although the occurrence of precocious sexual dimorphism is not uncommon in ostracodes, other authors (e.g. Szczechura et al., 1991 and Bassiouni, 2002) follow Majoran's separation of species.

$$
\text { Cythereis Jones, } 1849
$$

Type-species. Cytherina ciliata Reuss, 1846

Cythereis algeriana Bassoulet \& Damotte, 1969

(Figures 5E-F)

1969 Cythereis algeriana Bassoulet \& Damotte, p. 141, pl. 1, figs. a-d.

1981 Cythereis algeriana Bassoulet \& Damotte. Al-AbdulRazzaq \& Grosdidier, p. 181, pl. 1, fig. 4. 


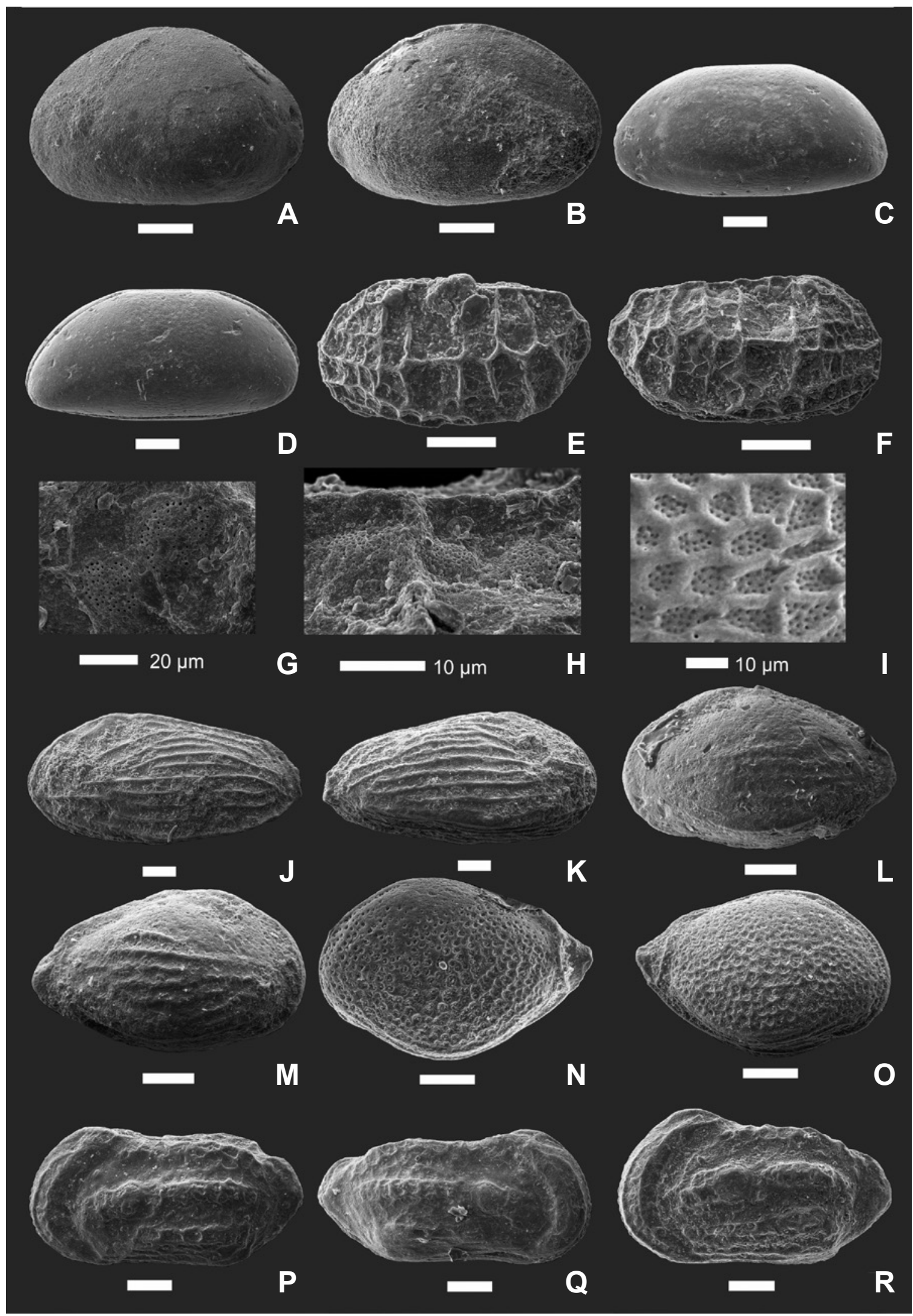

Figure 4. A-B, Xestoleberis sp. 1., A, C left view (MP-O-2221); B, same specimen right view. C-D, Xestoleberis sp. 2, C, C left view (MP-O-2222); D, same specimen right view. E-H, Aracajuia distincta, E, C left view (MP-O-2223); F, same specimen right view. G, detail of a posteromedian pore-cluster, H, detail of a posterodorsal pore-cluster. I, Xylocythere sp., LV (MP-O-2052), detail of pore-clusters. J-K, Metacytheropteron berbericus; J, C in right view (MP-O-2224); K, same specimen left view. L-M, Metacytheropteron sp., L, C left view (MP-O-2225); M, same specimen right view. N-O, Eocytheropteron retroversicardinatum, N, C left view (MP-O-2226), O, same specimen right view. P-R, Sergipella sp., $\mathbf{P}$, male C left view (MP-O-2228); $\mathbf{Q}$, same specimen right view; $\mathbf{R}$, female C left view (MP-O-2227). Scale bars $=100 \mu \mathrm{m}$, unless otherwise stated. 
Figured specimen. MP-O-2232 C, 1: $0.89 \mathrm{~mm}$, h: $0.49 \mathrm{~mm}$, w: $0.49 \mathrm{~mm}$.

Origin. Sample $80 \mathrm{~m}$.

Material. Six specimens.

Remarks. Cythereis algeriana was described in the late Cenomanian of Algeria.

Cythereis namousensis Bassoulet \& Damotte, 1969

(Figures G-H)

Figured specimen. MP-O-2233 C, 1: $0.70 \mathrm{~mm}$, h: $0.40 \mathrm{~mm}$, w: $0.44 \mathrm{~mm}$.

Origin. Sample $200 \mathrm{~m}$.

Material. Eight specimens.

Remarks. Cythereis namousensis was described in the late Cenomanian of Algeria.

\section{Cythereis sp. 1}

(Figures 5I-J)

1974 Veeniacythereis jezzineensis (Bischoff, 1963). Rosenfeld \& Raab, pl. 3, fig. 28.

Figured specimen. MP-O-2565 C, 1: $1.05 \mathrm{~mm}$, h: $0.56 \mathrm{~mm}$, w: $0.56 \mathrm{~mm}$.

Origin. Sample $170 \mathrm{~m}$.

Material. One specimen.

Remarks. The specimen figured by Rosenfeld \& Raab (1974) clearly differs from Veeniacythereis jezzinensis and is cospecific to Cythereis sp. 1.

\section{Cythereis sp. 2}

(Figures 5K-L)

Figured specimen. MP-O-2566 C, 1: $0.75 \mathrm{~mm}$, h: $0.44 \mathrm{~mm}$, w: $0.42 \mathrm{~mm}$.

Origin. Sample $70 \mathrm{~m}$.

Material. One specimen.

Aysegulina (Deroo, 1966) Özdikman, 2010

1966 Limburgina Deroo

Type species. Cypridina ornata Bosquet, 1847

$$
\text { Aysegulina sp. }
$$

(Figures 5M-N)

Figured specimen. MP-O-2567 C, 1: $0.86 \mathrm{~mm}$, h: $0.44 \mathrm{~mm}$, w: $0.49 \mathrm{~mm}$.

Origin. Sample $220 \mathrm{~m}$.

Material. One specimen.

Peloriops Al-Abdul-Razzaq, 1979a

Type species. Peloriops sphaeromata Al-Abdul-Razzaq, $1979 \mathrm{a}$
Peloriops ulosa Al-Abdul-Razzaq, 1979a

(Figures 5O-P)

1979a Peloriops ulosa Al-Abdul-Razzaq, p. 51, pl. 1, figs. 4-5, 11, 15.

1994 Peloriops ulosa Al-Abdul-Razzaq. Athersuch, pl. 12.2, fig. 4.

Figured specimen. MP-O-2568 C, 1: $0.82 \mathrm{~mm}$, h: $0.40 \mathrm{~mm}$, w: $0.30 \mathrm{~mm}$.

Origin. Sample $190 \mathrm{~m}$.

Material. Three specimens.

$$
\text { Glenocythere Al-Abdul-Razzaq, 1979b }
$$

Type species. Glenocythere bahreinsis Al-Abdul-Razzaq, 1979b

Glenocythere abdulrazzaqae Morsi \& Bauer, 2001

(Figures 5Q-R; 6A)

Figured specimens. MP-O-2569 female C, 1: $0.77 \mathrm{~mm}$, h: $0.49 \mathrm{~mm}, \mathrm{w}: 0.44 \mathrm{~mm}$; MP-O-2570 male C, 1: $0.96 \mathrm{~mm}$, h: $0.56 \mathrm{~mm}, \mathrm{w}: 0.54 \mathrm{~mm}$.

Origin. Sample $310 \mathrm{~m}$.

Material. Two specimens.

Remarks. This species was described in the Cenomanian deposits of Halal Formation (Sinai, Egypt).

Superfamily CYTHERIDEOIDEA Sars, 1925

Family PROGONOCYTHERIDAE Sylvester-Bradley, 1948

Neocythere Mertens, 1956

Type species. Neocythere vanveeni Mertens, 1956

Neocythere sp.

(Figures 6B-C)

Figured specimens. MP-O-2571 C, 1: $0.49 \mathrm{~mm}, \mathrm{~h}: 0.30 \mathrm{~mm}$, w: $0.30 \mathrm{~mm}$.

Origin. Sample $200 \mathrm{~m}$.

Material. Four specimens.

Family CYTHERIDEIDAE Sars, 1925

Perissocytheridea Stephenson, 1938

Type species. Cytheridea matsoni Stephenson, 1935

Perissocytheridea ignota Szczechura, Abd-Elshafy \& Babinot, 1991

(Figures 6D-F)

Figured specimens. MP-O-2572 female C, 1: $0.72 \mathrm{~mm}$, h: $0.40 \mathrm{~mm}, \mathrm{w}: 0.37 \mathrm{~mm}$; MP-O-2573 male C, $1: 0.68 \mathrm{~mm}$, h: $0.30 \mathrm{~mm}, \mathrm{w}: 0.30 \mathrm{~mm}$. 

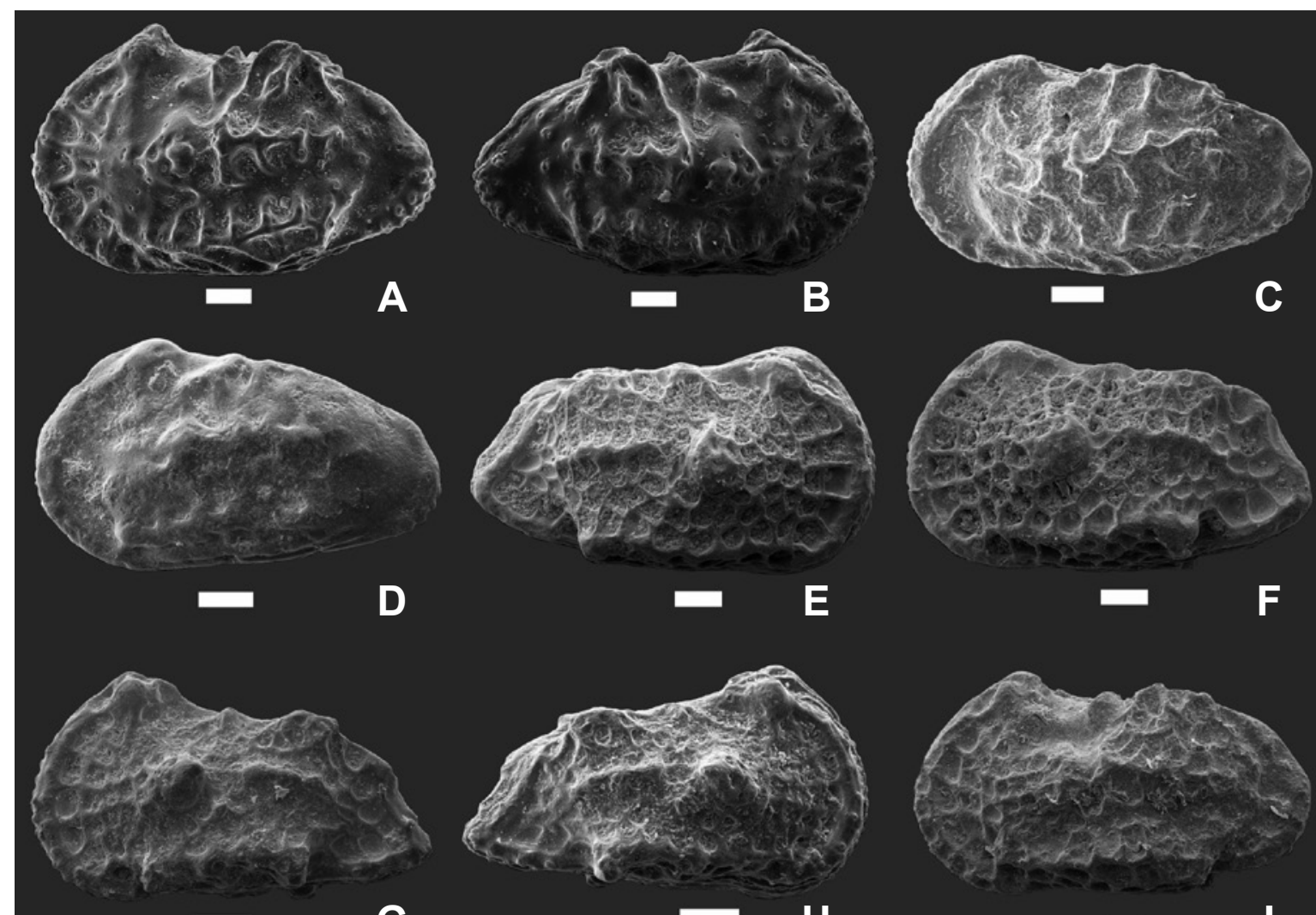

E

$\mathbf{F}$
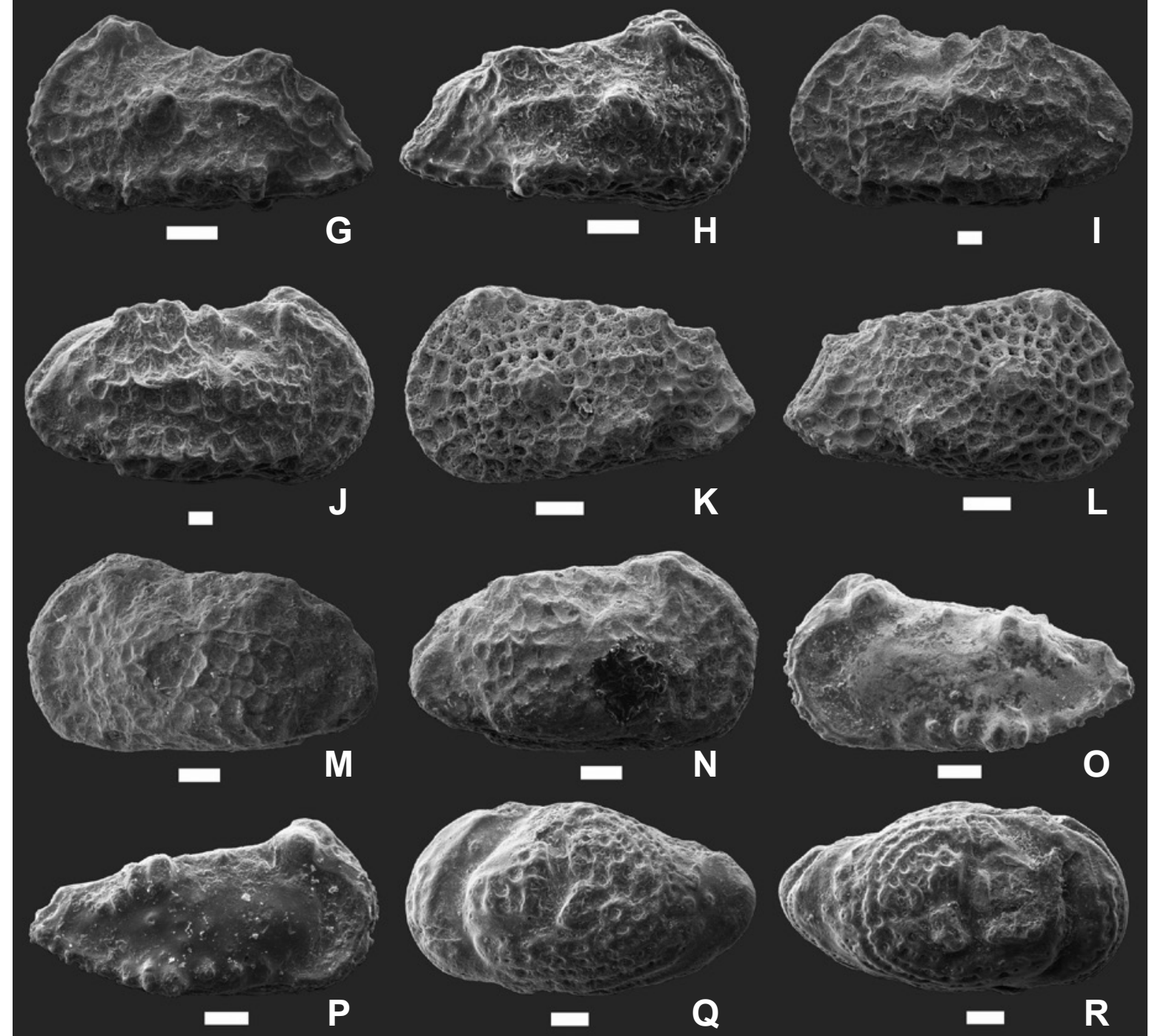

Q

$\mathbf{R}$

Figure 5. A-D, Veeniacythereis jezzineensis, A, C left view (MP-O-2229); B, same specimen right view; C, A-1(?) C left view (MP-O-2230); D, A-2(?) C left view (MP-O-2231). E-F, Cythereis algeriana, E, C left view (MP-O-2232); F, same specimen right view. G-H, Cythereis namousensis, G, C left view (MP-O-2233); H, same specimen right view. I-J, Cythereis sp. 1, I, C left view (MP-O-2565); J, same specimen right view. K-L, Cythereis sp. 2, K, C left view (MP-O-2566); L, same specimen right view. M-N, Aysegulina sp. M, C left view (MP-O-2567); N, same specimen right view. O-P, Peloriops ulosa, O, C left view (MP-O-2568); P, same specimen right view. Q-R, Glenocythere abdulrazzaqae, $\mathbf{Q}$, male C left view (MP-O-2570); $\mathbf{R}$, same specimen right view. Scale bars $=100 \mu \mathrm{m}$. 


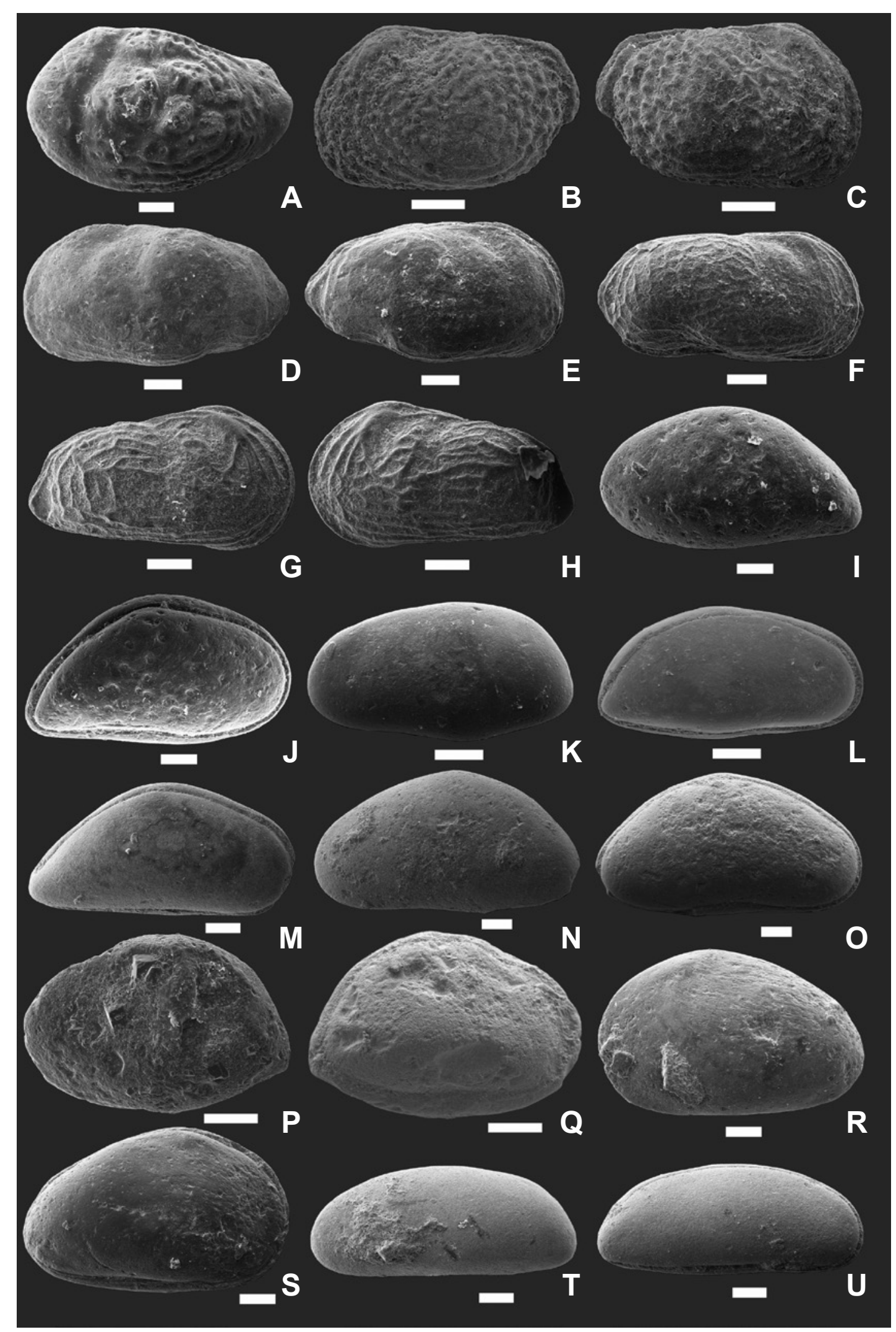

Figure 6. A, Glenocythere abdulrazzaqae, female C left view (MP-O-2569). B-C, Neocythere sp., B, C left view (MP-O-2571); C, same specimen right view. D-F, Perissocytheridea ignota, D, female C left view (MP-O-2572); E, same specimen right view; F, male C right view (MP-O-2573). G-H, Perissocytheridea sp., G, C left view (MP-O-2574); H, same specimen right view. I-M, Dolocytheridea atlasica, I, morphotype 1, C left view (MP-O-2575); J, same specimen right view; K, morphotype 2, C left view (MP-O-2576); L, same specimen right view; M, morphotype 3, C left view (MP-O-2577). N-O, Kalyptovalva tifratinensis, N, C left view (MP-O-2578); O, same specimen right view. P-Q, Dordoniella sp., P, C left view (MP-O-2579); Q, same specimen right view. R-S, Cytherideidae gen. et sp. indet., R, C left view (MP-O-2580); S, same specimen right view. T-U, Parakrithe sp., T, C left view (MP-O-2581); U, same specimen right view. Scale bars $=100 \mu \mathrm{m}$. 
Origin. Sample $260 \mathrm{~m}$.

Material. 260 specimens.

Remarks. This species was described in the Cenomanian deposits of the Northern Galala (Egypt). It is one of the most abundant species in the samples herein studied and occurs mostly in the basal part of the well NH-1.

\section{Perissocytheridea sp. \\ (Figures 6G-H)}

Figured specimen. MP-O-2574 C, 1: $0.61 \mathrm{~mm}, \mathrm{~h}: 0.33 \mathrm{~mm}$, w: $0.26 \mathrm{~mm}$.

Origin. Sample $200 \mathrm{~m}$.

Material. One specimen.

Remarks. Perissocytheridea sp. differs from P. ignota, being narrower and strongly reticulated. Due to its scarcity, it might constitute a caved species.

\section{Dolocytheridea Triebel, 1938}

Type species. Cytherina hilseana Roemer, 1841

Dolocytheridea atlasica Bassoulet \& Damotte, 1969

(Figures 6I-M)

1969 Dolocytheridea atlasica Bassoulet \& Damotte, pl. 2, figs. 9a-d.

1991 Dolocytheridea atlasica Bassoulet \& Damotte. Szczechura et al., pl. 3, fig. 4

2009 Dolocytheridea atlasica Bassoulet \& Damotte. Boukhary et al., p. 23, pl. 1, figs. 5-8 (see this for a more complete synonymy).

Figured specimens. MP-O-2575 morphotype $1 \mathrm{C}, 1: 0.75$ $\mathrm{mm}$, h: $0.40 \mathrm{~mm}$, w: $0.35 \mathrm{~mm}$; MP-O-2576 morphotype $2 \mathrm{C}$, 1: $0.56 \mathrm{~mm}, \mathrm{~h}: 0.30 \mathrm{~mm}, \mathrm{w}: 0.26 \mathrm{~mm}$; MP-O-2577 morphotype $3 \mathrm{C}, 1: 0.77 \mathrm{~mm}, \mathrm{~h}: 0.33 \mathrm{~mm}$, w: $0.29 \mathrm{~mm}$.

Origin. Sample $330 \mathrm{~m}$ (MP-O-2575), sample $80 \mathrm{~m}$ (MP-O2576), sample $140 \mathrm{~m}$ (MP-O-2577).

Material. 415 specimens.

Remarks. This is a typical species from cenomanian deposits from the South Tethys, described in the late Cenomanian of Algeria. According to some authors Dolocytheridea atlasica presents variability and three morphotypes were registered in the present study, designated as morphotypes 1,2 and 3. The occurrence of intraspecific variability is not uncommon in Cretaceous cytherideids (e.g. Fossocytheridea ventrotuberculata Bergue et al., 2011) and is, probably, environmentally induced.

\section{Kalyptovalva Howe \& Laurencich, 1958}

Type species. Cytheridea ovata Bosquet, 1854

Kalyptovalva tifratinensis Andreu-Boussut, 1991

(Figures 6N-O)
Figured specimen. MP-O-2578 C, 1: $0.89 \mathrm{~mm}, \mathrm{~h}: 0.47 \mathrm{~mm}$, w: $0.40 \mathrm{~mm}$.

Origin. Sample $220 \mathrm{~m}$.

Material. One specimen.

Dordoniella Apostolescu, 1955

Type species. Dordoniella strangulata Apostolescu, 1955

Dordoniella sp.

(Figures 6P-Q)

1991 Schuleridea baidarensis Damotte \& Saint-Marc. Szczechura et al., pl. 10, figs. 4a,b.

Figured specimen. MP-O-2579 C, 1: $0.49 \mathrm{~mm}, \mathrm{~h}: 0.33 \mathrm{~mm}$, w: $0.26 \mathrm{~mm}$.

Origin. Sample $330 \mathrm{~m}$.

Material. One specimen.

Gen. et sp. indet.

(Figures 6R-S)

Figured specimen. MP-O-2580 C, 1: $0.77 \mathrm{~mm}$, h: $0.47 \mathrm{~mm}$, w: $0.35 \mathrm{~mm}$.

Origin. Sample $60 \mathrm{~m}$.

Material. One specimen.

Family KRITHIDAE Mandelstam, 1958

Parakrithe Bold, 1958

Type species. Cytheridea (Dolocytheridea) vermunti Bold, 1946

Parakrithe sp.

(Figures 6T-U)

Figured specimen. MP-O-2581 C, 1: $0.79 \mathrm{~mm}$, h: $0.33 \mathrm{~mm}$, w: $0.35 \mathrm{~mm}$.

Origin. Sample $130 \mathrm{~m}$.

Material. One specimen.

\section{RESULTS AND DISCUSSION}

Thirty-nine ostracode species belonging to 10 families and 20 genera were recorded. Tracyleberididae (nine spp.), Candonidae (eight spp.), Cytherideidae (six spp.), Cytherellidae (five spp.) and Bairdiidae (four spp.) are the richest families and constitute most of the ostracodes recovered from the NH-1 well. The assemblages are composed of several species previously recorded in Cenomanian deposits of Middle East and North Africa, such as Metacytheropteron berbericus, Cytherella aegyptiensis, Cythereis namousensis, Veeniacythereis jezzineensis and Dolocytheridea atlasica. Two of them, $V$. jezzinensis and $D$. atlasica, are common 
Tethyan Cenomanian taxa with problematic taxonomy and for that reason they will be briefly discussed here.

Comprehensive discussions on Veeniacythereis jezzineensis were carried out by Rosenfeld \& Raab (1983) and Majoran (1988). Those authors proposed, respectively, lumping and splitting approaches to deal with this species. Dolocytheridea atlasica is another intriguing Cenomanian species presenting morphological variability, where different patterns of outline (subtriangular to elongate) and ornamentation (smooth to punctate) are observed (Szczechura et al., 1991). Three morphotypes are illustrated in the present study.

To supply additional contribution to the complex taxonomy of these two taxa is far beyond the objectives and possibilities of the present study, inasmuch as it would demand careful sampling (coring and/or outcrop sampling), rigorous age calibration, and detailed morphological analyses. Due to the stratigraphic significance of those taxa, such a revision would be very contributive for biostratigraphic purposes. Considering that the present study is based in cutting samples and the majority of the specimens studied are carapaces, we adopt the lumping proposal by Rosenfeld \& Raab (1983) for Veeniacythereis jezzineensis, since the splitting of those forms in different species was not possible in the material from the NH-1 well. In reference to Dolocytheridea atlasica, we also assume that the three morphotypes belong to the same species, although strong differences in outline are observed.

Despite the ubiquity of some species in the Maghreb and the Middle East there are also species with restricted occurrences. This can be exemplified by the richness of Bairdia (four spp.) where only B. youssefi could be identified. Among the cytherellids, Cytherella sp. 1 constitutes a very rare species in the NH-1 well and with no previous record in other studies. The presence of a median caudal process is a morphological pattern seen in Senonian representatives of the genus, and Cytherella sp. 1 might be a caved specimen. Other representatives of the family, however, in especial $C$. aegyptiensis present broader geographic occurrences and are very abundant in Naur Formation.

The occurrence of pore-clusters in Aracajuia distincta constitutes the first record of this morphologic feature in the genus. Similar groupings of pores - which have no relation to the porecanals - have already been found in Eucytherurinae, like Microceratina Swanson, and Xylocythere Maddocks \& Steineck. Cabral et al. (2014) recorded comparable structures in Limnocytheridae, which were referred as sieve plates. The pore-clusters in $A$. distincta are always grouped in pairs and their pattern is vaguely similar to the observed in Xylocythere (Mazzini \& Gliozzi, 2000, p. 143, figs. 1g-i). An image of the pore-clusters of Xylocythere sp. recorded by Bergue \& Coimbra (2008) in the Quaternary of Santos Basin, Brazil, is presented in the Figure 4 for comparison. The function of these pores is still unknown, though van Harten (1993) proposed a possible relation to nutrient absorption. Apparently, they are morphological features that allow the entrance or elimination of substances through the carapace, either in feeding or chemosensory processes.

\section{ACKNOWLEDGEMENTS}

The authors are indebted to D. Danielopol and to the late J.-P. Colin whose discussion, comments and bibliographic supply improved the early version of this manuscript. T.M. Puckett and I. Slipper are thanked for the careful revision of the text. The third author is grateful to $\mathrm{CNPq}$ (Conselho Nacional de Desenvolvimento Científico e Tecnológico) for the financial support (proc. 304453/2013-7).

\section{REFERENCES}

Al-Abdul-Razzaq, S.K. 1979a. Peloriops, a new ostracode genus from the Cretaceous of Kuwait. In: N. Krstic (ed.) Taxonomy, biostratigraphy and distribution of ostracodes, Serbian Geological Society, p. 47-54.

Al-Abdul-Razzaq, S.K. 1979b. Glenocythere, a new ostracode genus from the Ahmadi Formation (Cretaceous) of Kuwait. Journal of Paleontology, 53:920-930.

Al-Abdul-Razzaq, S.K. 1980. New Eocytheropteron species with reverse valve structure. Micropaleontology, 26:444-448.

Al-Abdul-Razzaq, S.K. \& Grosdidier, E. 1981. Ostracode index species from the Cenomanian of the South Shelf of the Tethys Sea. Bulletin des Centres de Recherche Exploration-Production Elf Aquitaine, 5:173-191.

Alexander, C.I. 1929. Ostracoda of the Cretaceous of north Texas. Bulletin of the University of Texas, 2907:1-137.

Andreu-Boussut, B. 1991. Les ostracodes du Cretacé moyen (Barremian a Turonien), le long d'une transversal Agadir-Nador (Maroc). Strata, 14:1-765.

Antonietto, L.S.; Abrahão, A.; Do Carmo, D.A. \& Meirelles, R.P. 2013. Taxonomy, biostratigraphy and paleozoogeography of Amphicytherura Butler and Jones, Aracajuia Krömmelbein and Dinglecythere Antonietto et al., n. gen. (Crustacea, Ostracoda). Marine Micropaleontology, 105:1-7. doi:101016/j. marmicro.2013.09.002.

Apostolescu, V. 1955. Un noveau genre d'ostracode du Cénomanien de Dordogne: Dordoniella strangulata n.g., n.sp. Cahiers de Géologie, 33:329-331.

Athersuch, J. 1988. The Biostratigraphy of Cretaceous Ostracods from Oman. In: T. Hanai; N. Ikeya \& K. Ishizaki (eds.) Evolutionary Biology of Ostracoda, Elsevier, p. 739-755.

Athersuch, J. 1994. The biostratigraphic significance of Cretaceous ostracods from the Arabian Gulf. In: M.D. Simmons (ed.) Micropalaeontology and Hydrocarbon Exploration in the Middle East, Chapman \& Hall, p. 253-272.

Babinot, J.F. \& Basha, S.H. 1985. Ostracodes from the early Cenomanian of Jordan. A preliminary report. Geobios, 18:257-262.

Babinot, J.F. \& Colin, J.-P. 1988. Paleobiogeography of Tethyan Cretaceous Marine Ostracods. In: T. Hanai; N. Ikeya \& K. Ishizaki (eds.) Evolutionary Biology of Ostracoda, Elsevier, p. 739-755.

Baird, W. 1838. The natural history of the British Entomostraca. Magazine of Zoology and Botany, 2:132-144.

Basha, S.H. 1985. Foraminifera and Ostracoda from Lower Cretaceous rocks of Jordan. Neues Jahrbuch fur Geologie und Paläontologie, 12:736-750.

Bassiouni, M.A.A. 2002. Middle Cretaceous (Aptian-Early Turonian) Ostracoda from Sinai, Egypt. Neue Palaontologische Abhandlungen, 4:1-122. 
Bassoulet, J.P. \& Damotte, R. 1969. Quelques ostracodes nouveaux du Cenomano-Turonien de l'Atlas Saharien occidental (Algerie). Revue de Micropaléontologie, 12:130-144.

Benson, R.H. \& Tatro, J.O. 1964. Faunal description of Ostracoda of the Marlbrook marl (Campanian), Arkansas. University of Kansas Paleontological Contributions, Arthropoda, 7:1-32.

Benson, R.H.; Berdan, J.M.; Bold, W.A.; Hanai, T.; Hessland, I.; Howe, H.V.; Kesling, R.V.; Levinson, S.A.; Reyment, R.A.; Moore, R.C.; Scott, H.W.; Shaver, R.H.; Sohn, I.G.; Stover, L.E.; Swain, F.M.; Sylvester-Bradley, P.C. \& Wainwright, J. 1961. In: R.C. Moore \& C.W. Pitrat (eds.) Treatise on Invertebrate Paleontology, part Q, Arthropoda 3, Crustacea, Ostracoda, Geological Society of America and University of Kansas Press, p. 99-421.

Bergue, C.T. \& Coimbra, J.C. 2008. Late Pleistocene and Holocene bathyal ostracodes from the Santos Basin, southeastern Brazil. Palaeontographica Abteilung A, 285:101-144.

Bergue, C.T.; Fauth, G.; Vieira, C.E.L.; Santos, A.S. \& Viviers, M.C. 2011. New species of Fossocytheridea Swain \& Brown, 1964 (Ostracoda, Crustacea) in the Upper Cretaceous of Santos Basin, Brazil. Revista Brasileira de Paleontologia, 14:149-152. doi:10.4072/rbp.2011.2.03.

Bischoff, G. 1963. Ostracoden-Studien im Libanon, 1. Die Gattung Cythereis in der Unterkreide. Senckenbergiana Lethaea, 44:1-77.

Bismuth, H.; Boltenhagen, C.; Donze, P.; Le Fevre, J \& SaintMarc, P. 1981. Le Cretacé moyen et supérieur du Djebel Semmama (Tunisie du Centre-Nord): microstratigraphie et évolution sédimentologique. Bulletin du Centre de Recherche, Exploration-Production Elf-Aquitaine, 5:193-267.

Bold, W. van den. 1946. Contribution to the study of Ostracoda with special reference to the Cretaceous and Tertiary microfauna of the Caribbean region. Utrecht University, Doctoral Thesis, $167 \mathrm{p}$.

Bosquet, J. 1847. Description des Entomostracés fossiles de la craie de Maestricht. Mémoires de la Société Royale des Sciences de Liége, 4:353-378.

Bosquet, J. 1854. Les Crustaces fossiles du terrain Cretace du Limbourg. Verhandelingen Uitgeven door de Commissie Belast met het Vervaardigen eener Geologische Beschrijvig en Kaart van Neederland, 2:53-126.

Boukhary, M.; Morsi, A.-M.M.; Eissa, R. \& Kerdany, M. 2009. Late Cenomanian ostracod faunas from the area south of Ain Sukhna, western side of the Gulf of Suez, Egypt. Geologia Croatica, 62:19-30.

Burdon, D. 1959. Handbook of the Geology of Jordan to accompany and explain the three sheets of the 1:250000 Geological Map East of the Rift by A. M. Quennell. Colchester, Benham \& Co., 82 p.

Cabral, M.C.; Danielopol, D.L.; Carbonel, P.; Gross, M.; Stoica, M.; Külköylüoglu, O.; Piller, W.; Yavuzatmaca, M.; Humphreys, W.F. 2014. Sieve-type pores on the valves of Timiriaseviinae. Their interst for systematics of selected taxa. In: 25ème RÉUNION DES OSTRACODOLOGISTES DE LANGUE FRANCAISE (ROLF), Abstracts, Université de Perpignan, 3 p.

Colin, J.-P. \& El Dakkak, M.W. 1975. Quelques ostracodes du Cénomanien du Djebel Nezzazat, Sinaï, Egypte. Revista Española de Micropaleontología, Special Volume:49-60.

Colin, J.-P; Platel, J.-P; Roger, J. \& Tambareau, Y. 2001. Nouvelles données sur les faunes d'ostracodes de l'Albien et du Cénomanien d'Oman: taxonomie et paléobiogéographie. In: R.H. Weiss (ed.) Contributions to Geology and Palaeontology of Gondwana, in honour of Helmut Wopfner, University of Cologne, p. 89-109.

Coryell, H.N.; Sample, C.H. \& Jennings, P.H. 1935. Bairdoppilata, a new genus of Ostracoda, with new species. American Museum Novitates, 777:1-5.
Damotte, R. \& Grosdidier, E. 1963. Quelques ostracodes du Crétacé inférieur de Champagne humide. 1. Albien-Cenomanien. Revue de Micropaleontologie, 6:51-66.

Damotte, R. \& Saint-Marc, P. 1972. Contribution a la conaissance des ostracodes Cretacé du Liban. Revista Española de Micropaleontología, 4:273-296.

Do Carmo, D.A.; Colin, J.-P.; Hidalgo, P.H.P.; Meireles, R.P.; Berbert-Born, M.L.C. \& Almeida, C.M. 2012. Reassessment of the genus Sergipella Krömmelbein, 1967 (Ostracoda, Trachyleberididae), uppermost Aptian-Albian of Brazil and West Africa: Taxonomy and paleogeographic distribution. Revue de Micropaléontologie, 55:3-15. doi:10.1016/j.revmic.2011.12.002

Gerry, E. \& Rosenfeld, R. 1973. Amphicytherura distincta and Neocyprideis vandenboldi (Ostracoda), new species from the Cenomanian-Turonian of Israel. Revista Española de Micropaleontología, 5:99-105.

Grosdidier, E. 1973. Associations d'ostracodes du Crétacé d'Iran. Revue de l'Institut Français du Pétrole, 28:131-170.

Gründel, J. 1968. Trachyleberididae (Ostracoda) aus den SalzmergelFazies (Santon) des westlichen Teils der DDR. Freiberger Forschungshefte (Paläontologie), 234:31-43.

Harten, D. van. 1993. Deep sea hydrothermal vent eucytherurine Ostracoda: the enigma of the pore-clusters and the paradox of the hinge. In: K.G. Mckenzie \& P.J. Jones (eds.) Ostracoda in the Earth and Life Sciences, Balkema Publishers, p. 571-580.

Honigstein, A. 1984. Senonian ostracodes from Israel. Bulletin of the Geological Survey of Israel, 78:1-48.

Krömmelbein, K. 1967. Ostracoden aus der marinen "KüstenKreide" Brasiliens - 2. Sergipella transatlantica n. g. n. sp., und Aracajuia benderi n. g. n. sp., aus dem Ober-Aptium/Albium. Senckenbergiana Lethaea, 48:525-533.

Liebau, A. 2005. A revised classification of the higher taxa of the Ostracoda (Crustacea). Hydrobiologia, 538:115-137. doi: 10.1007/s10750-004-4943-7.

Luger, P. 2003. Paleobiogeography of late Early Cretaceous to Early Paleocene marine Ostracod in Arabia and north to equatorial Africa. Palaeogeography, Palaeoclimatology, Palaeoecology, 196:319-342. doi:10.1016/S0031-0182(03)00462-2

Maddocks, R.F. 1969. Revision of Recent Bairdiidae. Bulletin of the United States National Museum, 295:1-126.

Majoran, S. 1988. Comments on a miscellaneous ostracod group from the Middle Cretaceous of the south shelf of Tethys Sea. Journal of African Earth Sciences, 6:691-702.

Majoran, S. 1989. Mid-Cretaceous Ostracoda of northeastern Algeria. Fossil and Strata, 27:1-67.

Majoran, S. 1996. Mid-Cretaceous "Veeniacythereis" (Ostracoda) from Africa and the Middle East. Neues Jahrbuch fur Geologie und Paläontologie, 3:183-192.

Mazzini, I. \& Gliozzi, E. 2000. Ocurrence of fossil and Recent Microceratina Swanson, 1980 (Ostracoda, Eucytherinae) in the Mediterranean. Micropaleontology, 46:143-152.

McCoy, F. 1844. Synopsis of the Characters of the Carboniferous Fossils of Ireland (Crustacea). Dublin Univ. Press, Dublin, p. 159-168.

Mertens, E. 1956. Zur Grenzziehung Alb/Cenoman in Nordwestdeutschland mit Hilfe von Ostracoden. Geologie Jahrbuch, 72:173-230.

Morsi, A.M. \& Bauer, J. 2001. Cenomanian ostracods from Sinai Peninsula, Egypt. Revue de Paleobiologie, 20:377-414.

Morsi, A.M. \& Wendler, J.E. 2010. Biostratigraphy, palaeoecology and palaeogeography of the Middle Cenomamian-Early Turonian Levant Platform in Central Jordan based on ostracods. In: 
C. Homberg \& M. Bachmann (eds.) Evolution of the Levant Margin and Western Arabia Platform since the Mesozoic, London, Geological Society of London, p. 187-210 (Special Publication 341).

Oertli, H.J. 1957. Ostracodes du Jurassique supérieur du Bassin de Paris (Sondage Vernon 1). Revue de l'Institut Français du Pétrole et Annales des Combustibles Liquides, 12:647-695.

Özdikman, H. 2010. Substitute names for three genera of Ostracoda (Crustacea). Munis Entomology and Zoology, 5:315-316.

Parker, D.H. 1970. The hydrogeology of the Mesozoic-Cenozoic aquifers of the western highland and plateaus of East Jordan. Rome, United Nations Development Program/Food and Agriculture Organization, 4 vols, 424 p. (Technical Report 212).

Powell, J.H. 1989. Stratigraphy and sedimentation of the Phanerozoic rocks in Central and south Jordan. Part B: Kurnub, Ajlun and Belqa groups. Bulletin Natural Resources Authority, 11:1-130.

Puckett, T.M.; Colin, J.-P. \& Mitchell, S. 2012. New species and genera of Ostracoda from the Maastrichtian (Late Cretaceous) of Jamaica. Micropaleontology, 58:397-455.

Reuss, A.E. 1846. Die Versteinerungen der Böhmischen Kreideformation. Schweizerbart, 2:59-148.

Roemer, F.A. 1841. Die Versteinerungen des norddeutschen Kreidegebirges. Hannover, 145 p.

Rosenfeld, A. 1981. Cytherella gigantosulcata (Ostracoda), a replacement for Cytherella sulcata Rosenfeld, 1974. Journal of Paleontology, 55:896.

Rosenfeld, A. \& Raab, M. 1974. Cenomanian-Turonian ostracodes from the Judea group in Israel. Bulletin of the Geological Survey of Israel, 62:1-64.
Rosenfeld, A. \& Raab, M. 1983. Ontogenesis and stratigraphy of the ostracod Veeniacythereis jezzineensis (Bischoff, 1963). Journal of Micropalaeontology, 2:59-65.

Rossetti, G. \& Martens, K. 1998. Taxonomic revision of the Recent and Holocene representatives of the family Darwinulidae (Crustacea, Ostracoda) with a description of three new genera. Bulletin de l'Institute Royal des Sciences Naturelles de Belgique, Biologie, 68:55-100.

Sars, G.O. 1866. Oversigt af Norges marine ostracoder. Förhandlinger i Videnskabs-Selskabet i Christiania, 1865:1-130.

Schneider, G.F. 1956. In.: A.V. Suzin (ed.) Ostrakody tretichnykh otlozheniy severnovo preddkavkazya, Moskow, $191 \mathrm{p}$.

Schultze, F.; Marzouk, A.M.; Bassiouni, M. \& Kus, J. 2004. The late Albian-Turonian carbonate platform succession of west-central Jordan: stratigraphy and crises. Cretaceous Research, 25:709-737. doi:10.1016/j.cretres.2004.06.008

Stephenson, M.B. 1935. Some microfossils of the Potamides matsoni Zone of Lousiana. Geological Bulletin of Lousiana Department of Conservation, 6:187-196.

Szczechura, J.; El Shafy, E. \& Babinot, J.F. 1991. Late Albian to Early/Mid-Cenomanian ostracodes from northern Galala Plateau, Egypt. Acta Palaeontologica Polonica, 36:3-38.

Viviére, J.-L. 1985. Les ostracodes du Crétacé supérieur (Vraconien à Campanien basal) de la région de Tebessa (Algérie du Nord(Est): stratigraphie, paléoécologique, systématique. Université Pierre et Marie Curie, Paris, Doctoral thesis, $261 \mathrm{p}$.

Received in December, 2015; accepted in May, 2016. 